\title{
Laboratory implementation of edge illumination X-ray phase-contrast imaging with energy-resolved detectors
}

\author{
P. C. Diemoz ${ }^{1, *}$, M. Endrizzi ${ }^{1}$, F. A. Vittoria ${ }^{1}$, C. K. Hagen ${ }^{1}$, M. Marenzana ${ }^{2}$, P. Delogu ${ }^{3}$, A. \\ Vincenzi $^{4}$, L. De Ruvo ${ }^{3}$, G. Spandre ${ }^{3,4}$, A. Brez ${ }^{3,4}$, R. Bellazzini ${ }^{3,4}$ and A. Olivo ${ }^{1}$ \\ ${ }^{I}$ Department of Medical Physics and Biomedical Engineering, University College London, United \\ Kingdom \\ ${ }^{2}$ Kennedy Institute of Rheumatology, University of Oxford, United Kingdom \\ ${ }^{3}$ Istituto Nazionale di Fisica Nucleare, Pisa Section, Pisa, Italy \\ ${ }^{4}$ PIXIRAD Imaging Counters s.r.l., c/o INFN Pisa, Italy
}

"Corresponding author:p.diemoz@ucl.ac.uk

\begin{abstract}
Edge illumination (EI) X-ray phase-contrast imaging (XPCI) has potential for applications in different fields of research, including materials science, non-destructive industrial testing, small-animal imaging, and medical imaging. One of its main advantages is the compatibility with laboratory equipment, in particular with conventional non-microfocal sources, which makes its exploitation in normal research laboratories possible. In this work, we demonstrate that the signal in laboratory implementations of EI can be correctly described with the use of the simplified geometrical optics. Besides enabling the derivation of simple expressions for the sensitivity and spatial resolution of a given EI setup, this model also highlights the EI's achromaticity. With the aim of improving image quality, as well as to take advantage of the fact that all energies in the spectrum contribute to the image contrast, we carried out EI acquisitions using a photon-counting energy-resolved detector. The obtained results demonstrate that this approach has great potential for future laboratory implementations of EI.
\end{abstract}

Keywords: edge illumination, X-ray imaging, phase contrast, energy-resolved detectors, geometrical optics

\section{MOTIVATION/PURPOSE}

The last two decades have seen a rising interest in X-ray phase-contrast imaging (XPCI) and, in particular in recent years, increasing efforts have been dedicated to its translation from synchrotron radiation (SR) facilities to laboratory implementations [1-8]. In XPCI, the image contrast is produced by the phase shift that X-rays experience by passing through the sample, in addition to the attenuation contrast exploited by conventional X-ray imaging techniques [8]. The advantage in terms of achievable image contrast is particularly important in the case of light materials, where the attenuation differences are small and the performance of conventional X-ray methods is thus intrinsically limited [9].

Several XPCI techniques have been developed, which differ in the physical mechanisms exploited for converting the Xray phase shifts into intensity modulations measurable with a detector [8]. In addition to the differences in the setup arrangement and required instrumentation, the various techniques are also characterized by different requirements in terms of coherence of the radiation illuminating the object, size of the X-ray source, detector spatial resolution, etc. Indeed, the strict experimental requirements generally imposed by XPCI methods have limited their application to very large and expensive SR facilities, or to microfocal X-ray tubes. The use of the latters, however, comes at the expense of much increased exposure times due to the limited flux, which is not acceptable for many applications, notably medicine and small-animal imaging. Strong effort is therefore dedicated to exploring new implementations that could make XPCI compatible with laboratory equipment, and in particular with commercially available non-microfocal X-ray tubes. This would greatly expand the spectrum of applications that could be tackled with XPCI, as it would enable its use in normal research laboratories and potentially in clinical facilities. 
Edge illumination (EI) XPCI is, in this respect, one of the most promising methods. Besides providing excellent image quality at SR facilities $[4,10,11]$, EI was in fact demonstrated to be efficiently applicable also to conventional X-ray tubes $[7,12,13]$. As discussed in more detail in the following, this is due to the incoherent nature of the method, which does not require neither spatial nor temporal coherence for the radiation illuminating the object. EI has already been successfully implemented in the imaging of samples from different fields of application, e.g. breast imaging [14], cartilage imaging [15], and security [16]. Recently, its laboratory application in combination with computed tomography was also demonstrated [17], which enables the visualization of the 3-dimensional structure of the sample. In this work, we focus on the modelling of the technique through the use of geometrical optics, which enables a simple estimation of crucial quantities such as phase sensitivity and spatial resolution, and highlights the independence from the X-ray energy. We also show the application of EI with an energy resolved photon-counting detector, which experimentally proves the achromatic nature of the method and, additionally, offers ways to potentially enhance the image quality.

\section{METHODS}

The main principles at the basis of EI are briefly presented in Fig. 1. The beam produced by an X-ray tube is split into a plurality of beamlets by an absorbing mask placed in front of the sample (the sample mask). In the typical implementation of EI featuring one-dimensional phase sensitivity, the collimation acts only in one direction ( $y$ in Fig. 1, as the apertures are assumed to extend in the direction $x$ entering the plane of the drawing). The size of the apertures usually ranges from a few $\mu \mathrm{m}$ to a few tens of $\mu \mathrm{m}$. A second absorption mask (the detector mask) is placed in front of the detector, so as to create insensitive regions between adjacent pixels. The two masks are misaligned, so that each beamlet straddles the edge of an aperture in the detector mask. Note that the period of the second mask is designed to match that of the detector, while the period of the sample mask is equal to the demagnified detector mask period. X-ray refraction occurs in the sample, especially at its interfaces where the phase shift $\phi(y)$ is varying more rapidly, as the refraction angle is equal to $\Delta \theta_{y}(y)=k^{-1} \partial \phi / \partial y(y)$, where $k=2 \pi / \lambda$ is the wave number and $\lambda$ the X-ray wavelength. As a result, the beamlet is physically deviated from its trajectory and its position on the detector mask is shifted by the quantity $z_{2} \Delta \theta_{y}$, where $z_{2}$ is the distance between the sample and the detector mask. The effect of this shift will be either an increase or a decrease in the counts on the detector, depending on the direction of refraction, as photons are deviated in or out of the sensitive region of the pixel (see Fig. 1). In a single EI measurement, the object is effectively sampled with a rate equal to the period of the sample mask, which thus determines the accessible spatial resolution. However, it was shown that the spatial resolution can be further increased if the so-called "dithering" is performed. This procedure consists in acquiring several images with the sample shifted in steps of a fraction of the period, and in recombining the various acquisitions to yield a more finely sampled image [12,18,19].

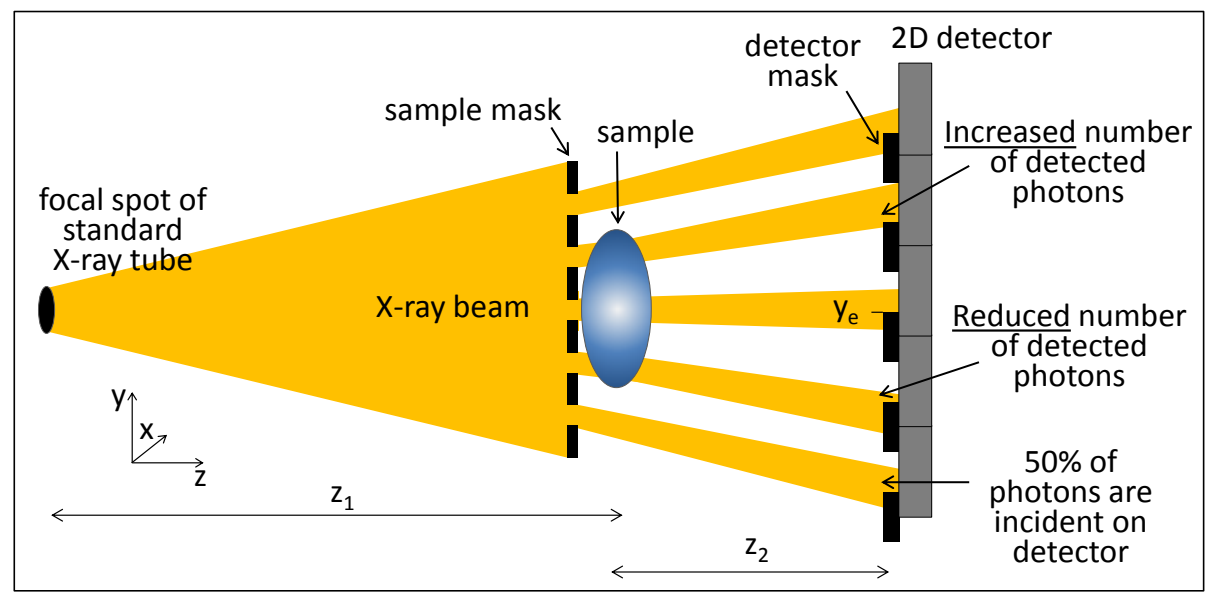

Figure 1: Schematic representation of the principles at the basis of EI (diagram not to scale).

More precisely, the intensity incident on the detector mask in the case of a monochromatic beam can be written as $[11,13]$ : 


$$
I_{o b j}(y ; p ; \lambda)=T(p ; \lambda) \cdot I_{r e f}\left(y-z_{2} \Delta \theta_{y}(p ; \lambda) ; \lambda\right)
$$

where $p$ indicates the position along $y$ of the considered sample aperture, $T$ is the object transmission, and $I_{r e f}(y ; \lambda)$ is the beam intensity on the detector mask that would be obtained without the sample. The signal recorded by the detector is then equal to the integral of the intensity within the corresponding detector aperture, i.e.:

$$
S_{\text {obj, mono }}\left(p ; y_{e} ; \lambda\right)=T(p ; \lambda) \int_{y_{e}}^{y_{e}+d} d y I_{r e f}\left(y-z_{2} \Delta \theta_{y}(p ; \lambda) ; \lambda\right)=S_{0}(\lambda) T(p ; \lambda) C\left(y_{e}-z_{2} \Delta \theta_{y}(p ; \lambda)\right)
$$

where $S_{0}(\lambda)=\int_{-\infty}^{+\infty} d y I_{r e f}(y ; \lambda), \quad y_{e}$ is the position of the lower edge of the detector aperture and $d$ its size. $C\left(y_{e} ; \lambda\right) \equiv S_{0}^{-1} \int_{y_{e}}^{y_{e}+d} d y I_{r e f}(y ; \lambda)$ is the so-called illumination curve, which describes how the signal varies as a function of the misalignment between the masks. It is comprised between a value close to 0 (when the absorbing septa of the detector mask are completely covering the beamlets), and a value close to 1 (when the sample and detector masks are perfectly aligned). Examples of $I_{r e f}$ and $C$ curves, simulated using wave optics calculations [20], are presented in Figs. $2 \mathrm{a}$ and $2 \mathrm{~b}$, respectively. An X-ray energy of $20 \mathrm{keV}$, and the experimental parameters of one of our experimental setups, have been considered: source-to-sample distance $z_{1}=1.6 \mathrm{~m}, z_{2}=0.4 \mathrm{~m}$, detector mask period $=98 \mu \mathrm{m}$, detector mask aperture $=29 \mu \mathrm{m}$, sample mask aperture $=23 \mu \mathrm{m}$, source full width at half maximum $=70 \mu \mathrm{m}$.
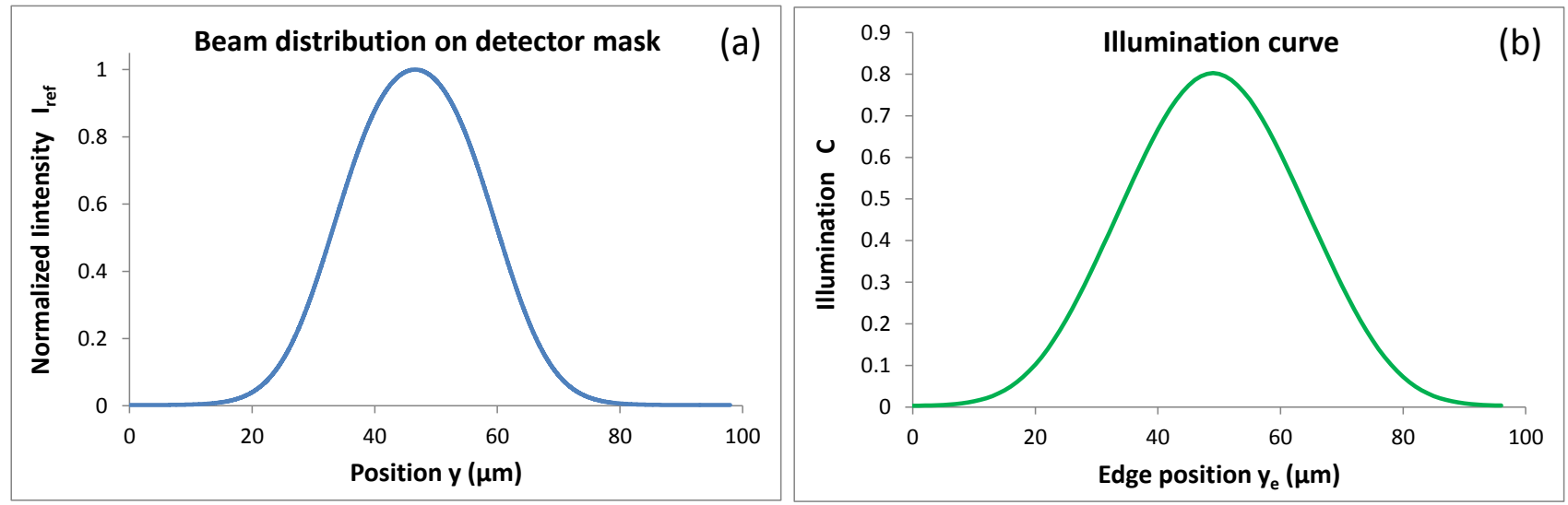

Figure 2: Simulated profiles for a) $I_{r e f}$ and b) $C$ (see text for details on the considered experimental parameters).

In the general case when a polychromatic beam is considered, the signal is the sum of all the monochromatic components [21]:

$$
S_{o b j, p o l y}\left(p ; y_{e}\right)=S_{0} \int_{\lambda_{\min }}^{\lambda_{\max }} d \lambda f(\lambda) T(p ; \lambda) C\left(y_{e}-z_{2} \cdot \Delta \theta_{y}(p ; \lambda) ; \lambda\right)
$$

where $S_{0}$ represents the signal that would be acquired in the absence of detector mask and sample, and $f(\lambda)$ the normalized energy spectrum incident on the sample. $I_{r e f}$ and $C$ are, in general, a function of the X-ray energy. This is due to two reasons: a) the diffraction of the wave through the propagation distance $z_{2}$ is dependent on the energy, which affects the shape of the beam at the detector plane, and b) in the case of limited mask thickness, the transmission through their absorbing region (ideally equal to zero) cannot be neglected and is energy dependent. However, we consider here that the masks are sufficiently thick so as to absorb all X-ray energies in the spectrum. Moreover, it can be demonstrated that, in the case of polychromatic beams emitted by conventional extended sources (leading to negligible coherence at the object plane), the diffraction ripples are washed out, so that the shape of the beam and the effect of the object can be correctly described by using simple geometrical optics $[13,22,23]$. This amounts to considering X-rays as travelling along straight lines, thus neglecting diffraction effects. Under these assumptions, the EI setup can be considered 
achromatic, as the only dependences upon energy are those of the object (i.e. attenuation and refraction). Note that, in this approximation, the illumination curve is simply given by the convolution of the detector aperture, of the magnified sample aperture, and of the projected source distribution.

It is important to notice that EI shares these incoherence properties with another XPCI technique, free-space propagation (FSP), when the latter is applied in the near-field regime [3]. This result is not accidental, but rather arises from the fact that the EI image contrast originates from free-space propagation between the sample and the detector mask. This strong connection between EI and FSP was formally demonstrated in recent work [23].

Under these assumptions, and in the simplified case where refraction angles are small, such that the illumination curve can be linearly approximated around $y_{e}$, Eq. 3 can be rewritten as:

$$
S_{o b j, p o l y}\left(p ; y_{e}\right) \approx C\left(y_{e}\right) S_{0} T_{e f f}(p) \cdot\left[1-C^{\prime}\left(y_{e}\right) C^{-1}\left(y_{e}\right) z_{2} \Delta \theta_{y, e f f}(p)\right]
$$

where

$$
T_{e f f}(p) \equiv \int_{\lambda_{\min }}^{\lambda_{\max }} d \lambda f(\lambda) T(p ; \lambda)
$$

and

$$
\Delta \theta_{y, e f f}(p) \equiv T_{e f f}^{-1}(p) \cdot \int_{\lambda_{\min }}^{\lambda_{\max }} d \lambda f(\lambda) T(p ; \lambda) \Delta \theta_{y}(p ; \lambda)
$$

are the effective transmission and refraction angle defined in the case of a polychromatic beam. Eqs. 4-6 show that the effect of polychromaticity can be simply taken into account by weighting $T$ and $\Delta \theta_{y}$ over the energy spectrum.

The contrast in a single EI image is determined by both the attenuation and refraction introduced by the sample (see Eqs. 3 and 4). However, it was shown in previous work that these two properties can be separated and quantified, if two images of the sample are acquired at different mask misalignments and then mathematically combined [11-13]. Typically, the two images are obtained using opposite mask misalignments, i.e. with the detector mask stopping either the lower or the upper part of the beam [11-13]. It was also shown that the uncertainty on the retrieved refraction angle can be estimated by propagating the statistical error in the two input images, which gives [13]:

$$
\alpha=\sigma\left(\Delta \theta_{y, e f f}\right)=\frac{\sqrt{C\left(y_{e}\right)}}{z_{2} C^{\prime}\left(y_{e}\right) \sqrt{2 T_{e f f} S_{0}}}
$$

The quantity $\alpha$ represents the smallest detectable refraction angle, and, therefore, the angular sensitivity of the setup. Equation 6 is a very useful result: it can be exploited to study the influence of the various experimental parameters on the image sensitivity, which can be especially helpful for the design and optimization of new setups.

The modelling of EI through geometrical optics also enables the derivation of a simple expression describing the spatial resolution of a given setup, i.e. the size of the smallest detectable feature in the sample. In the case when dithering is not performed, the spatial resolution is equal to the period of the sample mask. However, if fine dithering steps are used, smaller features can be accessed. It can be shown, in fact, that the width of the point spread function (PSF) for the refraction signal is given by $[19,23]$ :

$$
P S F \text { width }=\min \left\{a, 4 \sigma_{r}\right\}
$$

where $a$ is the size of the sample aperture, $\sigma_{r}=z_{2}\left(z_{2}+z_{1}\right)^{-1} \sigma_{s}$ is the size of the source rescaled to the object plane and $\sigma_{s}$ the standard deviation of the source intensity distribution. The width of the refraction signal PSF represents the limit to the spatial resolution achievable in a given EI setup, when the dithering step is made very small. Equations 7 and 8 can be used in the design of a new EI setup, in order to optimize the experimental parameters for maximizing the sensitivity 
and spatial resolution, as done in [24]. Importantly, it was also shown that the optimal parameters for the two quantities do not generally match, thus making a compromise between sensitivity and spatial resolution necessary.

\section{RESULTS}

In this work, we have combined the laboratory implementation of EI with the capabilities of PIXIRAD, a photoncounting, energy-resolved detector [25]. The aim is two-fold: a) to improve image quality thanks to the virtually negligible dark noise of the detector and $b$ ) to take advantage of the additional energy information, to further enhance the detection of the sample features. PIXIRAD is based on a thin $(650 \mu \mathrm{m})$ pixelated CdTe crystal, coupled to a CMOS readout ASIC [25]. Thanks to the ability of thresholding each event, the detector can simultaneously produce images at different X-ray energies (with energy bins of $2 \mathrm{keV}$ ), when illuminated with a polychromatic beam.

In Fig. 3, we report experimental illumination curves acquired using a polychromatic beam, at different energy levels of the detector (in this case, the energy bins have been merged two-by-two, so that the final energy resolution is equal to 4 $\mathrm{keV})$. The employed setup uses distances $z_{1}=1.6 \mathrm{~m}$ and $z_{2}=0.4 \mathrm{~m}$, sample mask aperture $a=23 \mu \mathrm{m}$ and detector mask aperture $d=29 \mu \mathrm{m}$. The source is a Rigaku M007, employing a Molybdenum target and operated at $40 \mathrm{kVp}, 20 \mathrm{~mA}$. The obtained plots demonstrate the achromaticity of the considered EI setup, since the illumination curve shows very little dependence upon the energy, as expected. The very small offset visible in all curves also proves that the two masks are sufficiently thick and are therefore fully absorbing at the considered energies.

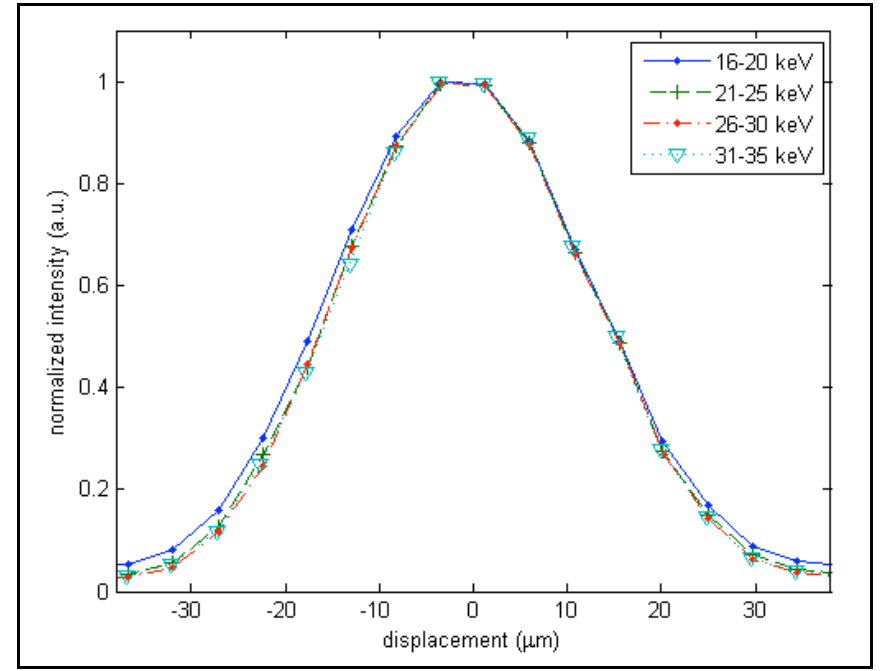

Figure 3: Illumination curves at different $X$-ray energies obtained using a polychromatic beam and the PIXIRAD detector (see text for details on the experimental parameters).

In Figs. $4 a$ and $4 b$, we present retrieved transmission and refraction images of a rabbit tibia. The high image quality achieved enables the clear visualization of the fine structures in the sample. The superiority of the refraction image, in terms of the level of detail observable, is also apparent. In this preliminary study, we simply summed the images obtained at the different energy bins, thus not exploiting the energy resolving capabilities of the detector. However, alternative ways to combine and process the images acquired at different energies will be considered and analyzed in future work, for example along the lines described in [26], with the aim of optimizing the contrast-to-noise and the image quality. 

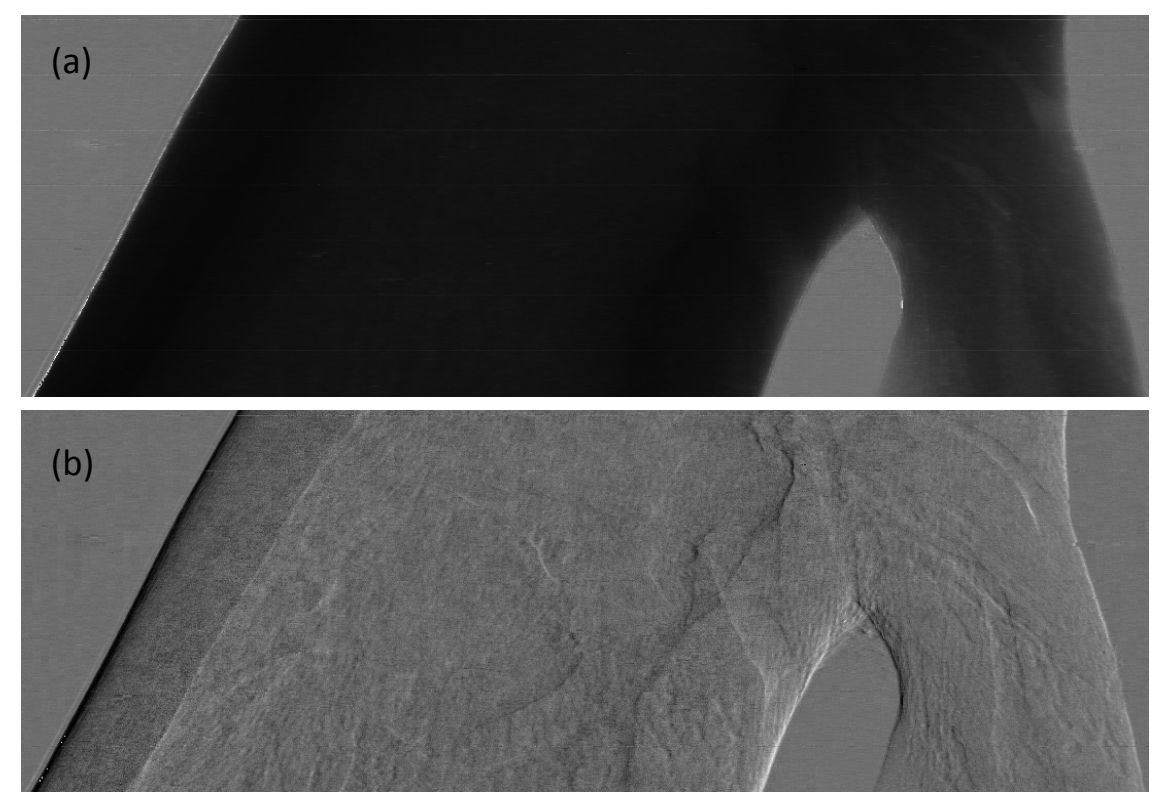

Figure 4: (a) Transmission and (b) refraction angle images of a rabbit tibia obtained using the PIXIRAD detector.

\section{CONCLUSIONS}

In this work, we show that the EI XPCI method, when implemented with conventional non-microfocal sources, can be modelled through the use of geometrical optics. Among the main consequences of this is the achromaticity of the EI setup, as the only dependence upon X-ray energy is due to the object attenuation and refraction properties. This means that all energies in the spectrum contribute to the signal, and can thus be exploited to produce the image contrast. We take full advantage of this property by using an EI laboratory setup in combination with PIXIRAD, a photon-counting energy-resolved detector. Besides enhancing the image quality thanks to its excellent noise properties, PIXIRAD allows measurement and discrimination of the various energy components. This additional energy information will prove useful in the future to improve the detection of the sample features as well as for the quantitative characterization of the sample.

This work is not being and has not been submitted for publication or presentation elsewhere.

\section{REFERENCES}

[1] A. Snigirev, I. Snigireva, V. Kohn, S. Kuznetsov, and I. Schelokov, "On the possibility of X-ray phase contrast microimaging by coherent high-energy synchrotron radiation,” Rev. Sci. Instrum. 66(12), 5486-5492 (1995).

[2] V. N. Ingal and E. A. Beliaevskaya, "X-ray plane-wave topography observation of the phase contrast from a non-crystalline object,” J. Phys. D: Appl. Phys. 28, 595 (1995).

[3] S. W. Wilkins, T. E. Gureyev, D. Gao, A. Pogany, and A. W. Stevenson, "Phase-contrast imaging using polychromatic hard X-rays," Nature 384(6607), 335-338 (1996).

[4] A. Olivo et al., "An innovative digital imaging set-up allowing a low-dose approach to phase contrast applications in the medical field," Med. Phys. 28(8), 1610-1619 (2001).

[5] A. Momose, S. Kawamoto, I. Koyama, Y. Hamaishi, K. Takai, and Y. Suzuki, "Demonstration of X-ray Talbot interferometry," Jpn. J. Appl. Phys. 42(Part 2, No. 7B), L866-L868 (2003).

[6] F. Pfeiffer, T. Weitkamp, O. Bunk, and C. David, "Phase retrieval and differential phase-contrast imaging with low-brilliance X-ray sources," Nat. Phys. 2(4), 258-261 (2006).

[7] A. Olivo and R. D. Speller, "A coded-aperture technique allowing X-ray phase contrast imaging with conventional sources," Appl. Phys. Lett. 91(7), 074106 (2007).

[8] S. W. Wilkins, Y. I. Nesterets, T. E. Gureyev, S. C. Mayo, A. Pogany, and A. W. Stevenson, "On the evolution and relative merits of hard X-ray phase-contrast imaging methods," Phil. Trans. R. Soc. A 372, 20130021 (2014).

[9] A. Bravin, P. Coan, and P. Suortti, "X-ray phase-contrast imaging: from pre-clinical applications towards clinics," Phys. Med. Biol. 58(1), R1-R35 (2013). 
[10] A. Olivo, P. C. Diemoz, and A. Bravin, "Amplification of the phase contrast signal at very high x-ray energies," Opt. Lett. 37(5), 915-917 (2012).

[11] P. C. Diemoz, M. Endrizzi, C. E. Zapata, Z. D. Pešić, C. Rau, A. Bravin, I. K. Robinson, and A. Olivo, "X-ray phase-contrast imaging with nanoradian angular resolution,” Phys. Rev. Lett. 110(13), 138105 (2013).

[12] P. R. T. Munro, K. Ignatyev, R. D. Speller, and A. Olivo, "Phase and absorption retrieval using incoherent X-ray sources," Proc. Natl. Acad. Sci. U.S.A. 109(35), 13922-13927 (2012).

[13] P. C. Diemoz, C. K. Hagen, M. Endrizzi, and A. Olivo, "Sensitivity of laboratory based implementations of edge illumination X-ray phase-contrast imaging," Appl. Phys. Lett. 103(24), 244104 (2013).

[14] A. Olivo et al., "Low-dose phase contrast mammography with conventional X-ray sources," Med. Phys. 40(9), 090701 (2013).

[15] M. Marenzana, C. K. Hagen, P. Das Neves Borges, M. Endrizzi, M. B. Szafraniec, K. Ignatyev, and A. Olivo, "Visualization of small lesions in rat cartilage by means of laboratory-based X-ray phase contrast imaging," Phys. Med. Biol. 57(24), 81738184 (2012).

[16] K. Ignatyev, P. R. T. Munro, D. Chana, R. D. Speller, and A. Olivo, "Coded apertures allow high-energy x-ray phase contrast imaging with laboratory sources", J. Appl. Phys. 110, 014906 (2011).

[17] C. K. Hagen, P. R. T. Munro, M. Endrizzi, P. C. Diemoz, and A. Olivo, "Low-dose phase contrast tomography with conventional x-ray sources," Med. Phys. 41(7), 070701 (2014).

[18] K. Ignatyev, P. R. T. Munro, R. D. Speller, and A. Olivo, "Effects of signal diffusion on x-ray phase contrast images," Rev. Sci. Instrum. 82(7), 073702 (2011).

[19] P. C. Diemoz, F. A. Vittoria, and A. Olivo, "Spatial resolution of edge illumination X-ray phase-contrast imaging," Opt. Express 22(13), 15514-29 (2014).

[20] F. A. Vittoria, P. C. Diemoz, M. Endrizzi, L. Rigon, F. C. Lopez, D. Dreossi, P. R. T. Munro, and A. Olivo, "Strategies for efficient and fast wave optics simulation of coded-aperture and other x-ray phase-contrast imaging methods," Appl. Opt. 52(28), 6940-6947 (2013)

[21] A. Pogany, D. Gao, and S. W. Wilkins, "Contrast and resolution in imaging with a microfocus x-ray source," Rev. Sci. Instrum. 68(7), 2774-2782 (1997).

[22] P. R. T. Munro, K. Ignatyev, R. D. Speller, and A. Olivo, "The relationship between wave and geometrical optics models of coded aperture type X-ray phase contrast imaging systems," Opt. Express 18(5), 4103-4117 (2010).

[23] P. C. Diemoz and A. Olivo, "On the origin of contrast in edge illumination X-ray phase-contrast imaging," Opt. Express 22(23), 28199-28214 (2014).

[24] P. C. Diemoz, M. Endrizzi, C. K. Hagen, T. P. Millard, F. A. Vittoria, and A. Olivo "Angular sensitivity and spatial resolution in edge illumination X-ray phase-contrast imaging,” Nucl. Instr. Meth. Phys. Res. A, in press.

[25] R. Bellazzini, G. Spandre, A. Brez, M. Minuti, M. Pinchera and P. Mozzo, "Chromatic x-ray imaging with a fine pitch CdTe sensor coupled to a large area photon counting pixel ASIC," J. Instrum. 8, C02028 (2013).

[26] M. Das and Z. Liang, "Spectral x-ray phase contrast imaging for single-shot retrieval of absorption, phase, and differentialphase imagery," Opt. Lett. 39(21), 6343-6346 (2014). 